\title{
BMJ Open Dead space closure with quilting suture versus conventional closure with drainage for the prevention of seroma after mastectomy for breast cancer (QUISERMAS): protocol for a multicentre randomised controlled trial
}

\author{
Lobna Ouldamer, ${ }^{1,2}$ Julia Bonastre, ${ }^{3,4}$ Solène Brunet-Houdard, ${ }^{5,6}$ Gilles Body, ${ }^{1,5}$ \\ Bruno Giraudeau, ${ }^{5,7}$ Agnès Caille ${ }^{5,7}$
}

To cite: Ouldamer $\mathrm{L}$, Bonastre J, BrunetHoudard S, et al. Dead space closure with quilting suture versus conventional closure with drainage for the prevention of seroma after mastectomy for breast cancer (QUISERMAS): protocol for a multicentre randomised controlled trial. BMJ Open 2016;6: 009903.

doi:10.1136/bmjopen-2015009903

- Prepublication history for this paper is available online. To view these files please visit the journal online (http://dx.doi.org/10.1136/ bmjopen-2015-009903)

Received 10 September 2015 Revised 20 October 2015 Accepted 21 October 2015

CrossMark

For numbered affiliations see end of article.

Correspondence to Dr Lobna Ouldamer; I.ouldamer@chu-tours.fr

\section{ABSTRACT}

Introduction: Postoperative wound seroma is common after mastectomy. This complication is associated with significant impact on patient outcomes and healthcare costs. The optimal closure approach for seroma prevention remains unknown but some evidence suggests that quilting suture of the dead space could lower the incidence of seroma. The aim of this trial is to compare seroma formation using quilting suture versus conventional closure with drainage in patients undergoing mastectomy.

Methods and analysis: This is a multicentre, superiority, randomised controlled trial in women undergoing mastectomy with or without axillary involvement. Exclusion criteria include indication of bilateral mastectomy or immediate reconstruction and any physical or psychiatric condition that could impair patient's ability to cooperate with postoperative data collection or that do not allow an informed consent. 320 participants will be randomised in a 1:1 ratio to receive either quilting suture or conventional wound closure with drain. The primary outcome is seroma requiring either aspiration or surgical intervention within 21 days following mastectomy. Secondary outcomes include seroma regardless of whether or not it requires an intervention, surgical site infection, pain score, cosmetic result, patient's quality of life, costs and cost-effectiveness. The primary analysis will be an intention-to treat analysis performed with a $\chi^{2}$ test (or Fisher's exact test).

Ethics and dissemination: Written informed consent will be obtained from all participants. This study was approved by Tours Research ethics committee (CPP TOURS-Region Centre-Ouest 1 , 2014-R20, 16 December 2014). Study findings will be published in peer-reviewed journals and presented at relevant national and international breast cancer conferences.

Trial registration number: NCT02263651.
Strengths and limitations of this study

- QUISERMAS is the first multicentre randomised controlled trial to assess quilting suture of the dead space after mastectomy on seroma prevention.

- Surgeons and patients cannot be blinded to the surgical arm. Consequently, to reduce the risk of bias, we decided to consider for primary outcome only seroma requiring aspiration or surgical intervention.

- Cosmetic results will be assessed by an independent adjudication committee.

- An economic evaluation will be conducted alongside the trial.

\section{INTRODUCTION}

Breast cancer is the most common cancer worldwide. Surgical treatment is the preferred option and about 14000 mastectomies are performed each year in France. $^{1}$ Postoperative seroma is a common complication after mastectomy. ${ }^{2-9}$ This complication is secondary to the disruption of lymphatic channels that inevitably complicates extensive surgical dissection and disruption of tissue planes creating a dead space. Excessive fluid accumulation in a seroma stretches the skin, resulting in patient discomfort, impaired ipsilateral shoulder function and higher risk of surgical site infection. In rare cases, a fibrous encapsulated seroma forms that is resistant to conservative treatment and requires subsequent surgical resection. Thus, this complication may also impact healthcare costs requiring prolongation of hospital stay or unplanned outpatient visits and may delay adjuvant therapy. 
Conventional wound closure commonly uses suction drain after mastectomy to prevent seroma despite seroma frequently occurs after drain removal. ${ }^{10}$ Studies on seroma prevention have focused on the obliteration of the dead space through fibrinogen, thrombin sealants, glues or tetracyclin with poor results. ${ }^{11-20}$ The comparator in these studies was almost always conventional wound closure with suction drains as it is the most common practice. Some recent evidence suggests that quilting suture reduces the incidence of seroma. ${ }^{21-23}$ Quilting suture consists in suturing the skin flaps to the underlying musculature to reduce 'dead space'. ${ }^{24}$ It aims to restore the integrity of tissue planes. Ten Wolde et $a l^{22}$ retrospectively analysed 176 patients (87 who underwent conventional closure and 89 quilted patients) from 2 consecutive groups who underwent mastectomy and/or axillary lymph node dissection (ALND), this also included patients undergoing an ALND with lumpectomy in whom only the axilla was quilted. All patients had a drain in the pectoral area that was removed on the day of discharge, at least within $36 \mathrm{~h}$ following surgery. The incidence of seroma decreased significantly from $80.5 \%$ to $22.5 \%$ in the quilted group, $\mathrm{p}<0.01$ and the volume of aspirations from 1660 to $611 \mathrm{~mL}$ $(\mathrm{p}=0.05)$. Quilting closure technique was also assessed in an observational study based on 119 consecutive patients in our tertiary breast cancer unit whose 59 received quilting suture (without drain) and 60 received conventional closure with drainage. The results showed a significant reduction in seroma for patients with quilting suture as compared to patients with conventional closure with drain $\mathrm{OR}=0.26,95 \%$ CI 0.08 to 0.86 ; $\mathrm{p}=0.03$ ). The hypothesis around quilting efficacy is that dead space is the major contributor to seroma formation, and that this surgical technique applied to obliterate the dead space might reduce the incidence of this complication. ${ }^{23}$ As recommended in the IDEAL framework describing the stages for development of innovation in surgery, quilting suture now needs to be assessed in a controlled randomised trial. ${ }^{25}$ Thus, the aim of our project is to assess, in a randomised controlled trial, quilting suture of the dead space without drainage at the pectoral area as compared to conventional closure with drainage on seroma prevention within 21 days following mastectomy for breast cancer.

\section{STUDY OBJECTIVES}

Our primary objective is to assess the impact of quilting on rates of wound seroma requiring aspiration or surgical intervention within 21 days following mastectomy.

Secondary objectives are to compare quilting suture of the dead space without drainage of the pectoral area to conventional closure with drainage after mastectomy for breast cancer regarding wound-related complications, surgical morbidity, pain, shoulder movement, cosmetic results, health related quality of life, costs and cost-effectiveness.

\section{METHODS AND ANALYSIS}

\section{Study design}

QUISERMAS is a multicentre, superiority, randomised controlled trial with parallel groups comparing quilting suture with conventional closure with drain in the prevention of seroma in patients undergoing mastectomy with or without axillary surgery.

\section{Setting}

The trial is ongoing at the time of publication in four French university hospitals (Tours, Nantes, Poitiers and Rennes). The study is conducted in the breast surgery departments of these academic centres.

\section{PARTICIPANTS}

\section{Inclusion criteria}

The inclusion criteria are: (1) Female patients with operable breast cancer (invasive carcinoma and/or ductal carcinoma in situ) for whom mastectomy is recommended or preferred by the patient either alone or in association with axillary clearance either sentinel lymph node biopsy or standard level I/II axillary node dissection, (2) Age $\geq 18$ and $\leq 85$ years.

\section{Exclusion criteria}

The exclusion criteria are: (1) Patients with an indication of bilateral mastectomy or immediate reconstruction, (2) Planned outpatient surgery, (3) Patients with known degenerative neuromuscular disease with thoracic muscular damage, (4) Patients with any physical or psychiatric condition that could impair with outcome assessment and intended follow-up.

Study participants are patients who meet the selection criteria and are willing and able to sign written informed consent.

\section{Recruitment}

The first patient was randomised on October 2014. Enrolment is ongoing at the time of publication.

The recruitment process is planned to fit with routine practice. Potential participants to the trial are identified at the time they attend for diagnosis and treatment choice for their breast cancer in one of the four involved tertiary-care centres. Patients who meet selection criteria receive a brief study presentation and full participant information sheet by a clinician. After selection criteria confirmation and answering to potential further patient questions about the trial, written informed consent is obtained before surgery by the patient's surgeon.

Baseline data are collected following consent during the preoperative period.

\section{Randomisation}

Randomisation is undertaken by the surgeon (investigator) via a centralised secure web-based randomisation system. Randomisation in a 1:1 ratio is computer 
generated by an independent statistician from the INSERM CIC 1415 statistical unit. The allocation sequence is generated with a random permuted block design. Varying block sizes will not be revealed to ensure concealment. To avoid prognostic imbalance between the two groups, randomisation is stratified by recruiting centre and planned surgical procedure, either (A) mastectomy without axillary surgery, (B) mastectomy with sentinel lymph node biopsy or (C) mastectomy with standard level I/II axillary node dissection.

\section{Study interventions}

Mastectomies are performed by experienced breast surgeons using a standardised technique. The skin incision must include the tumour biopsy site, any invaded or oedematous skin, plus the nipple-areola complex. For dissecting the upper and lower skin flaps, finding the bloodless plane between the smaller lobules of the subcutaneous fat, and the larger lobules of the fat in the breast proper is required. Finally, the whole of the posterior aspect of the breast from the pectoralis major is freed. This study addresses the type of wound closure in mastectomy. So, only wound closure will differ between the two groups.

\section{Quilting suture}

In the quilting suture group, the skin flaps are sutured to the underlying pectoralis major with multiple parallel rows of $0 / 0$ vicryl or equivalent. Running sutures at periodic intervals $(<2 \mathrm{~cm})$ are placed from the skin flaps to the underlying muscle. Minor dimpling is considered acceptable and is expected to resolve. If severe dimpling is observed, stitches are removed and replaced. Efficiency of quilting suture relies on a rigorous repartition of the sutures with a special attention taken to the obliteration of the largest potential dead spaces and the empty axillary apex. The skin edges are sutured in the same way as for the control group. Closed suction is not used for draining the pectoral area.

\section{Conventional closure with drain}

In the conventional closure with drain group, the skin flaps are not fixed subcutaneously but sutured at the edges, a closed suction drain is inserted under the flaps in the dead space created by the dissection at the pectoral area. The drain is stitched to the skin. The skin is closed in two layers with absorbable sutures, a deep layer of 2.0 or 3.0 vicryl sutures or equivalent, and a subcuticular closure with absorbable 3.0 or 4.0 Monocryl sutures or equivalent. The drain is connected to a single suction bottle, which is changed every day, and the daily drain volume is monitored. The drain is removed on the day of discharge either when drain volume is less than $50 \mathrm{~mL}$ over $24 \mathrm{~h}$, regardless of time elapsed after surgery or at 5 days following surgery. Conventional closure with drain was chosen as the comparator group as it is the current practice in the centres where the study is conducted and more generally in European countries. ${ }^{10}$
If an axillary lymph node dissection is required in any group (quilting suture or conventional closure), skin incisions performed for the mastectomy are used. After the insertion of a suction drain, the axillary area is closed with vicryl sutures to create a separation with the dead space, quilted or not, at the pectoral area. The drain is connected to a single suction bottle which is changed every day and the daily drain volume is monitored. The axillary drain is removed on the day of discharge either when drain volume is less than $50 \mathrm{~mL}$ over $24 \mathrm{~h}$ regardless of time elapsed after surgery or at 5 days following surgery. Consequently, patients with axillary lymph node dissection have two drains and two suction bottles in the conventional closure group and only one axillary drain and one suction bottle in the quilting suture group.

\section{Surgeon expertise and intervention standardisation}

The licensed French doctors who are involved in this trial as practitioners have all been certified by the French ministry of health, have at least 1 year of surgical experience (senior with at least 1 year of fellowship validated), and will have taken a course to ensure that they adhere strictly to the study protocol and are familiar with quilting suture. To standardise quilting suture across centres and surgeons, a training period of at least 2 months is required as recommended in the Randomised Trials of Non pharmacologic Treatment extension of CONSORT Statement. ${ }^{26}$

\section{Study outcomes}

\section{Primary outcome}

The primary outcome is wound seroma requiring aspiration or surgical evacuation within 21 days following mastectomy. A seroma is defined as a postoperative fluid collection via palpation on clinical examination. The Common Terminology Criteria for Adverse Events (CTCAE) 4.0 which is a descriptive terminology used for adverse event reporting, provides a grading scale for seromas (lymphoceles): (1) grade 1: asymptomatic, clinical or diagnostic observation only, intervention not indicated, (2) grade 2: symptomatic, medical intervention indicated, (3) grade 3: severe symptoms, radiological endoscopic or elective operative intervention indicated. Only grade 2 and 3 seromas, that is, seromas requiring one or more aspirations or a surgical intervention will be considered as primary outcome.

This outcome was chosen as the primary outcome for three reasons. First, this outcome measure was the most used as primary outcome in reported published trials evaluating and comparing the efficacy of different methods in reducing the incidence of seromas when drainage was not used for all patients. ${ }^{27-31}$ It reflects both patient morbidity and additional medical costs. Focusing on seromas requiring interventions (aspiration or surgical intervention) is a more objective criterion than the simple presence of seroma (on physical examination or ultrasound finding). This allows to take into 
account only seromas having important consequences for the patient, indeed some authors discovered that $92 \%$ of their patients had seromas noted on ultrasound, but only less than half $(42 \%)$ required aspiration of the seroma. ${ }^{32} 33$ Second, we did not wish to use the total inpatient drainage volume as a primary outcome, because it implies to use suction drains in dead space in both study groups. Using such a drain at the pectoral area while quilting the dead space is not the innovative technique we wished to test because we believe that drains themselves encourage drainage by stimulating tissue reactions or by suction. Moreover, even if we used suction drains in both groups, the patients will not be blinded because quilting suture technique is responsible of minor skin dimpling effect expected to resolve which does not exist with the conventional closure technique. Finally, the only outcome that could be blind assessed is the cosmetic result by an adjudication committee. However, this outcome is not as medically relevant as seroma requiring intervention. We therefore chose to study the cosmetic result as a secondary outcome.

In most cases, a patient will return to her initial centre if an aspiration or surgical intervention for wound seroma is needed. These interventions will be collected in the patient medical records. Nevertheless, each patient will be asked, at day 21 visit about seroma and the need for aspiration or intervention since hospital discharge. In rare cases where patients will mention seroma requiring aspiration or intervention in another centre or by their family practitioner, a physician will be contacted (either by phone or email) to validate the patient report (the same procedure will be performed for other wound-related complications).

\section{Secondary outcomes}

Secondary outcomes include:

1. Wound-related complications:

- Wound seromas that necessitate aspiration or surgical intervention within 9 months following mastectomy.

- For each patient presenting a seroma that necessitates aspiration, the total volume of aspiration and number of aspirations will be recorded.

- Wound seroma whatever the grade at day 21 and 9 months after surgery.

- Other wound-related complications such as haematoma, skin flap necrosis, surgical site infection at day 21 and 9 months after surgery.

2. Surgical morbidity: Duration of the surgical procedure and intraoperative blood loss, length of hospital stay after surgery (days), number of outpatient visits (related to mastectomy) needed following participant's discharge within the 9 months follow-up.

3. Pain: Patient self-reported pain measured with the visual analogue scale pain scoring system from 0 (no pain) to 10 (unbearable pain) recorded before surgery, daily during hospitalisation and at 21 days and 9 months after surgery.

4. Shoulder movement: The range of arm movement scored from 1 to 4 according to estimated angles of arm abduction as 1 (less than $\left.90^{\circ}\right), 2\left(90-134^{\circ}\right), 3$ $\left(135-179^{\circ}\right)$ and $4\left(180^{\circ}\right)$. It will be measured by the surgeon before surgery and also at 21 days and 9 months after surgery.

5. Cosmetic results: Both patient and surgeon assessments of the cosmetic results will be documented at day 21 and 9 months after surgery, with possible response categories as follows: poor, acceptable, good and excellent. Digital photographs of the mastectomy area will be taken with standardised angles of incidence at 9 months. Results will be rated at the end of the study, by an adjudication committee blinded to treatment allocation in order to obtain a blinded surgical cosmetic-assessment.

6. Health-related quality of life: the EuroQoL-5D (EQ-5D)-5 L will be collected at baseline, 21 days and 9 months visits. The EQ-5D-5 L is an update of the $3 \mathrm{~L}$ version. It still consists of two pages-the EQ-5D-5 L descriptive system and the EQ visual analogue scale. The descriptive system comprises the same five dimensions as the EQ-5D-5D-3 L (mobility, self-care, usual activities, pain/discomfort, anxiety/ depression). However, each dimension now has five levels: no problems, slight problems, moderate problems, severe problems and extreme problems. The respondent is asked to indicate his/her health state by ticking in the box against the most appropriate statement in each of the five dimensions. This decision results in a 1-digit number expressing the level selected for that dimension. The digits for five dimensions can be combined in a 5-digit number describing the respondent's health state.

7. Direct medical costs and cost-effectiveness.

\section{Follow-up}

During follow-up, patients will receive usual care. All patients are followed for a 9-month period, with follow-up visit at 21 days and 9 months following surgery. Those visits fit in with routine follow-up after mastectomy in the participating centres. Schedule of enrolment, intervention and assessments are presented in table 1 .

Participant retention is promoted through the eligibility criteria (exclusion of patients with any physical or psychiatric condition that could impair with outcome assessment and intended follow-up). Moreover, loss to follow is unexpected because of the nature of the disease and relatively short follow-up, that is, 9 months.

\section{BLINDING}

It is not possible to blind patients or surgeons in our trial because of the nature of the studied intervention, surgical intervention that depends on care provider, as for a large part of other non-pharmacological 
Table 1 Schedule of enrolment, intervention and assessments

\begin{tabular}{|c|c|c|c|c|c|}
\hline Timepoint & $\begin{array}{l}\text { Baseline } \\
\text { (inclusion) }\end{array}$ & $\begin{array}{l}\text { Surgery } \\
\text { Day } 0\end{array}$ & $\begin{array}{l}\text { Hospitalisation } \\
\text { daily }\end{array}$ & $\begin{array}{l}\text { Follow-up } \\
\text { Day } 21 \\
\text { ( } \pm 5 \text { days) }\end{array}$ & $\begin{array}{l}\text { Follow-up } \\
9 \text { months } \\
\text { ( } \pm 15 \text { days) }\end{array}$ \\
\hline \multicolumn{6}{|l|}{ Enrolment } \\
\hline Eligibility screen & $\mathrm{X}$ & & & & \\
\hline Informed consent & $\mathrm{X}$ & & & & \\
\hline Clinical examination & $\mathrm{X}$ & & $\mathrm{X}$ & $\mathrm{X}$ & $\mathrm{X}$ \\
\hline $\begin{array}{l}\text { Previous medical examination } \\
\text { (mammogram, breast ultrasound, breast MRI } \\
\text { if required) }\end{array}$ & $x$ & & & & \\
\hline $\begin{array}{l}\text { Randomisation (as close as possible to the } \\
\text { surgery) }\end{array}$ & & $x$ & & & \\
\hline \multicolumn{6}{|l|}{ Intervention } \\
\hline Quilting suture & & $\mathrm{X}$ & & & \\
\hline Conventional closure with drain & & $\mathrm{X}$ & & & \\
\hline \multicolumn{6}{|l|}{ Assessments } \\
\hline Wound seroma evaluation & & & $\mathrm{X}$ & $\mathrm{x}$ & $\mathrm{X}$ \\
\hline Other wound complications & & & $\mathrm{X}$ & $\mathrm{X}$ & $\mathrm{X}$ \\
\hline Pain score & $\mathrm{X}$ & & $\begin{array}{l}\text { Day } 1 \text { after surgery only, } \\
1 \text { st evaluation after 6:00 }\end{array}$ & $x$ & $\mathrm{X}$ \\
\hline Photographs (for cosmesis assessment) & & & & & $\mathrm{X}$ \\
\hline Range of arm movement & $\mathrm{X}$ & & & $\mathrm{X}$ & $\mathrm{X}$ \\
\hline EQ-5D-5 L questionnaire and cost evaluation & $\mathrm{X}$ & & & $\mathrm{X}$ & $x$ \\
\hline $\begin{array}{l}\text { Patient/surgeon reported cosmesis } \\
\text { assessment }\end{array}$ & & & & $x$ & $x$ \\
\hline Adverse events & & & $x$ & $x$ & $x$ \\
\hline
\end{tabular}

interventions. ${ }^{34} 35$ Moreover, blinding of outcome assessor is not feasible for the primary outcome: seroma that require aspiration or surgical evacuation within 21 days following mastectomy. Indeed, after discharge, patients can visit at any time (in emergency or not) for a seroma or another postoperative complication. It is not possible to ensure that the clinician who will examine the patient is not the same as the surgeon who operated this patient. Moreover, as the patients cannot be blinded to the treatment allocation, it is difficult to ensure that they will not disclose it to the surgeon (outcome assessor of the trial). An adjudication committee blinded to treatment allocation aiming to a posteriori validates the indication of aspiration or surgical evacuation of a seroma is not relevant in this study because the decision depends on criteria that cannot be assessed retrospectively by photographs and medical records only.

\section{DATA MANAGEMENT}

Data is recorded on study specific case report forms (CRFs) via an electronic data capture system (CS Online). To maintain participant's anonymity, CRFs are identified only by a coded patient number and initials. All records that contain patient names or other identifying information will be stored separately from the study records and can be identified only by the coded patient number and initials. A data manager from the INSERM CIC 1415 biometry unit verifies the data and sends queries for missing or inconsistent data.

\section{SAMPLE SIZE}

The study sample size is based on a comparison of quilting suture versus conventional wound closure with drainage on seroma prevention. In our observational study data, $22 \%(n=13 / 60)$ of patients undergoing mastectomy with conventional wound suture developed a seroma that required aspiration or surgical intervention within 21 days following surgery. Owing to the multicentre profile of our study, the rate of seroma could be greater. We thus assume a rate of $30 \%$ in the control group. In the quilting suture, we expect to observe a rate of patients developing a seroma of $15 \%$. With these assumptions, a two-sided type I error of $5 \%$ and $90 \%$ power, a sample size of 160 patients per group is needed. Therefore, we plan to enrol a total of 320 patients.

To recruit this number of patients a 24-month inclusion period is anticipated.

\section{Statistical analyses}

The statistical analyses will follow an intention-to-treat approach. Analyses will be conducted using two-sided significance tests at the $5 \%$ significance level. A participant flow diagram will be reported. Group characteristics at baseline will be studied with descriptive statistics. No statistical tests will be performed on baseline characteristics.

The primary outcome will be assessed as a rate, defined as the number of patients who experienced a 
seroma requiring aspiration or surgical intervention within 21 days following mastectomy divided by the number of patients randomised into this group. To compare the incidence rates between the two randomised groups, we will use a $\chi^{2}$ test or Fisher's exact test, as appropriate.

Giving the patient profile, loss to follow-up is very unlikely. Generally, patients continue their follow-up in their original centre even if they move. However, if the case does occur, imputation of missing outcomes will be performed at least in a sensitivity analysis.

For secondary analysis, qualitative outcomes such as other postoperative wound-related complications, cosmetic results and shoulder movement will also be compared between the two arms using a $\chi^{2}$ test or a Fisher's exact test. The duration of the surgical procedure, length of hospital stay, intraoperative blood loss, will be compared using Wilcoxon tests or Student $\mathrm{t}$ tests, as appropriate. Repeated measures such as pain evaluation and health-related quality of life will be analysed using linear mixed-effects models to take into account the correlation of repeated measures from a given subject.

\section{Economic evaluation}

A cost-effectiveness study will be performed on the basis of resource use and HRQOL data collected alongside the trial.

Direct medical costs will be assessed from the hospital and the payer perspectives in both groups and during the whole follow-up period, that is, 9 months after surgery. For each patient, we will collect the healthcare resource use both in the hospital setting and primary care services. This covers the initial surgical stay (duration of the surgical procedure, number of consumables (drains and sutures), length of stay), subsequent hospital stays due to complications/infections, general practitioners and gynaecologist visits (over a 21-day period following surgery only) and home nursing care visits (over a 21-day period only).

To value resources, we will use the following unit costs information:

- Hospital stays: diagnosis-related group payment per discharge in the French prospective payment scheme.

- Visits: general fee classification (Nomenclature Générale des Actes Professionnels) and the reimbursement rate at the date of analysis.

Health states will be valued into utility coefficients using data from the EuroQoL group (European value set). It will allow computing QALYs for each patient in both groups.

Costs and QALYs will be compared between the two groups using non-parametric tests. Means and 95\% CIs for costs, QALYS and incremental net monetary benefit will be estimated using the non-parametric bootstrap method. Differences in costs and differences in QALYs observed in the bootstrap replicates will be represented in the cost-effectiveness plane. A cost-effectiveness acceptability curve will be computed.

\section{Monitoring}

No Data Monitoring Committee was formed because of the short duration of patient participation and known minimal risks for both arms. We did not plan any interim analysis. Adverse events will be collected and reported accordingly using the usual reported system of the sponsor.

\section{Ethics and dissemination}

In conformity with the Declaration of Helsinki, all participants will sign a written informed consent form that describes this study and provides sufficient information for patients to make an informed decision about their participation. Consent will be obtained from patients before they undergo any study procedure. Participants may withdraw from the study at any time during the clinical trial without any impact on their care. In that event, data collected prior to participant withdrawal will be used in the trial analysis except if a participant requests removal of all her data from the database. Sponsor of the study may audit trial conduct as deemed appropriate. A formal amendment to the local research ethics committee will be required for any amendments to the study protocol which may impact the conduct of the study, or the potential safety of or benefits to patients will require, if needed an amendment will also be required from the National regulatory Agency for Security of Medicines and healthcare products. Any protocol amendments will be communicated to investigators and oversight authority but also to trial participants and registries, if deemed necessary. The chief investigator will be given an access to the cleaned data set.

Reports will follow international guidelines: CONSORT Statement and Extension of the CONSORT Statement to Randomised Trials of Non pharmacologic Treatment. Research findings will be submitted for publication in peer-reviewed journals regardless of whether or not they are statistically significant. Authors will be individuals who have made key contributions to study design and conduct. Trial findings will also be submitted for presentation at scientific meetings. The study findings will also be presented at relevant national and international breast cancer conferences.

\section{DISCUSSION}

Previous reports in the literature have addressed the effect of quilting versus conventional closure with drainage after mastectomy for breast cancer on patient outcome. However, the studies reported to date are limited by small sample sizes, absence of randomisation, concomitant use of drainage with quilting suture and most studies were single centre initiatives that lacked sufficient power to inform surgical practice. Breast cancer surgeons appear to currently favour conventional wound closure with drainage, although current evidence suggests better patient outcomes with quilting suture. The QUISERMAS trial will aim to resolve these controversies 
by establishing the effectiveness of each method of mastectomy closure. This will have important clinical implications, as each wound closure type is easily applicable and already performed by breast cancer surgeons. A key limitation of the QUISERMAS trial is that surgeons and patients cannot be blinded to the surgical arms. This leaves the assessment of outcomes and decisions to intervent on seroma vulnerable to bias. A strength of our study is that it is designed to be a feasible, comparative effectiveness trial design that is similar to common clinical situations. Additionally, this clinical trial protocol was conducted to conform strictly to the CONSORT statement. The results of the QUISERMAS trial will be an important contribution in breast cancer surgery literature and are likely to lead changes in mastectomy closure. We expect that this study will provide the clinical basis and evidence that is required to perform quilting suture in routine when performing mastectomies.

\section{Author affiliations}

${ }^{1}$ Department of Gynecology, CHRU de Tours, Tours, France

${ }^{2}$ INSERM unit 1069, Tours, France

${ }^{3}$ Gustave Roussy, Service de Biostatistique et d'Epidemiologie, Villejuif, France ${ }^{4} \mathrm{CESP}$, Centre for Research in Epidemiology and Population Health, Villejuif, France

${ }^{5}$ Université François-Rabelais de Tours, PRES Centre-Val de Loire Université, Tours, France

${ }^{6} \mathrm{CHRU}$ de Tours, Unité d'Evaluation Médico-Economique, Tours, France ${ }^{7} \mathrm{CHRU}$ de Tours, Tours, France

Acknowledgements The authors acknowledge Carine Coffre (data manager), Aurélie Darmaillacq (clinical research associate) and Rachel Fontenay (health economist) for their constructive support during preparation and conduct of the trial. The authors would also like to express appreciation for the contributions from patients with breast cancer who will participate in this trial.

Contributors $\mathrm{LO}$ and $\mathrm{AC}$ helped to conceive and design the trial and wrote the manuscript. JB, BG and GB helped to conceive the trial and revised the manuscript. $\mathrm{LO}$ and $\mathrm{GB}$ will be investigators and will recruit patients and conduct the trial. AC planned the statistical analysis. $L O$ and $A C$ will supervise the trial. All authors read and approved the final manuscript.

Funding This trial is supported by a grant from the French Ministry of Health (PHRC 2013). The funding source has no role in the design of this trial and will not have any role during its execution, analyses, interpretation of the data, or decision to submit results.

Competing interests None declared.

Ethics approval Tours Research Ethics Committee (CPP TOURS-Region Centre-Ouest 1, 2014-R20, 16 December 2014).

Provenance and peer review Not commissioned; peer reviewed for ethical and funding approval prior to submission.

Open Access This is an Open Access article distributed in accordance with the Creative Commons Attribution Non Commercial (CC BY-NC 4.0) license, which permits others to distribute, remix, adapt, build upon this work noncommercially, and license their derivative works on different terms, provided the original work is properly cited and the use is non-commercial. See: http:// creativecommons.org/licenses/by-nc/4.0/

\section{REFERENCES}

1. $\mathrm{HCL}$, Invs, INCa, Francim, Inserm. Projections de l'incidence et de la mortalité par cancer en France en 2010. Rapport technique 2012.
2. Tejler G, Aspegren K. Complications and hospital stay after surgery for breast cancer: a prospective study of 385 patients. Br J Surg 1985;72:542-4.

3. Tadych K, Donegan WL. Postmastectomy seromas and wound drainage. Surg Gynecol Obstet 1987;165:483-7.

4. Bryant M, Baum M. Postoperative seroma following mastectomy and axillary dissection. Br J Surg 1987;74:1187.

5. Aitken DR, Hunsaker R, James AG. Prevention of seromas following mastectomy and axillary dissection. Surg Gynecol Obstet 1984;158:327-30.

6. Hayes JA, Bryan RM. Wound healing following mastectomy. Aust $N$ Z J Surg 1984;54:25-7.

7. Boostrom SY, Throckmorton AD, Boughey JC, et al. Incidence of clinically significant seroma after breast and axillary surgery. $J$ Am Coll Surg 2009;208:148-50.

8. Agrawal A, Ayantunde AA, Cheung KL. Concepts of seroma formation and prevention in breast cancer surgery. ANZ J Surg 2006;76:1088-95.

9. Pogson CJ, Adwani A, Ebbs SR. Seroma following breast cancer surgery. Eur J Surg Oncol 2003;29:711-17.

10. Barton A, Blitz M, Callahan D, et al. Early removal of postmastectomy drains is not beneficial: results from a halted randomized controlled trial. Am J Surg 2006;191:652-6.

11. Kuroi K, Shimozuma K, Taguchi T, et al. Evidence-based risk factors for seroma formation in breast surgery. Jpn J Clin Oncol 2006;36:197-206.

12. Schwabegger AH, Ninkovic MM, Anderl H. Fibrin glue to prevent seroma formation. Plast Reconstr Surg 1998;101:1744.

13. Saltz R, Sierra D, Feldman D, et al. Experimental and clinical applications of fibrin glue. Plast Reconstr Surg 1991;88:1005-15; discussion 1016-7.

14. Harada RN, Pressler VM, McNamara JJ. Fibrin glue reduces seroma formation in the rat after mastectomy. Surg Gynecol Obstet 1992;175:450-4.

15. Sanders RP, Goodman NC, Amiss LR Jr, et al. Effect of fibrinogen and thrombin concentrations on mastectomy seroma prevention. J Surg Res 1996;61:65-70.

16. Kulber DA, Bacilious N, Peters ED, et al. The use of fibrin sealant in the prevention of seromas. Plast Reconstr Surg 1997;99:842-9; discussion 850-1.

17. Butler CE. Treatment of refractory donor-site seromas with percutaneous instillation of fibrin sealant. Plast Reconstr Surg 2006;117:976-85.

18. Jain PK, Sowdi R, Anderson ADG, et al. Randomized clinical trial investigating the use of drains and fibrin sealant following surgery for breast cancer. Br J Surg 2004;91:54-60.

19. Taghizadeh R, Shoaib T, Hart AM, et al. Triamcinolone reduces seroma re-accumulation in the extended latissimus dorsi donor site. $J$ Plast Reconstr Aesthet Surg 2008;61:636-42.

20. Rice DC, Morris SM, Sarr MG, et al. Intraoperative topical tetracycline sclerotherapy following mastectomy: a prospective, randomized trial. J Surg Oncol 2000;73:224-7.

21. Kuroi K, Shimozuma K, Taguchi T, et al. Effect of mechanical closure of dead space on seroma formation after breast surgery. Breast Cancer 2006;13:260-5.

22. Ten Wolde B, Van Den Wildenberg FJ, Keemers-Gels ME, et al. Quilting prevents seroma formation following breast cancer surgery: closing the dead space by quilting prevents seroma following axillary lymph node dissection and mastectomy. Ann Surg Oncol 2014;21:802-7.

23. Ouldamer L, Caille A, Giraudeau B, et al. Quilting suture of mastectomy dead space compared with conventional closure with drain. Ann Surg Oncol 2015;22:4233-40.

24. Ouldamer L, Trefoux-Bourdet A, Duquesne M, et al. [How I do ... quilting suture of dead space after mastectomy]. Gynecol Obstet Fertil 2011;39:663-4.

25. Ergina PL, Barkun JS, McCulloch P, et al. IDEAL group. IDEAL framework for surgical innovation 2: observational studies in the exploration and assessment stages. BMJ 2013;346:f3011.

26. Boutron I, Moher D, Altman DG, et al. Extending the CONSORT statement to randomized trials of nonpharmacologic treatment: explanation and elaboration. Ann Intern Med 2008;148: 295-309.

27. Daltrey I, Thomson $\mathrm{H}$, Hussien $\mathrm{M}$, et al. Randomized clinical trial of the effect of quilting latissimus dorsi flap donor site on seroma formation. Br J Surg 2006;93:825-30.

28. Gisquet H, Delay E, Paradol PO, et al. [Prevention of seroma by quilting suture after harvesting latissimus dorsi flap. The "Chippendale" technic]. Ann Chir Plast Esthet 2010;55:97-103.

29. Dancey AL, Cheema M, Thomas SS. A prospective randomized trial of the efficacy of marginal quilting sutures and fibrin sealant in 
reducing the incidence of seromas in the extended latissimus dorsi donor site. Plast Reconstr Surg 2010;125:1309-17.

30. Sakkary MA. The value of mastectomy flap fixation in reducing fluid drainage and seroma formation in breast cancer patients. World $J$ Surg Oncol 2012;10:8

31. Gonzalez EA, Saltzstein EC, Riedner CS, et al. Seroma formation following breast cancer surgery. Breast J 2003;9:385-8.

32. Jeffrey SS, Goodson WH 3rd, Ikeda DM, et al. Axillary lymphadenectomy for breast cancer without axillary drainage. Arch Surg 1995;130:909-12; discussion 912-3.
33. Soon PSH, Clark J, Magarey CJ. Seroma formation after axillary lymphadenectomy with and without the use of drains. Breast 2005;14:103-7.

34. Boutron I, Tubach F, Giraudeau B, et al. Blinding was judged more difficult to achieve and maintain in nonpharmacologic than pharmacologic trials. J Clin Epidemiol 2004;57:543-50.

35. Jacquier I, Boutron I, Moher D, et al. The reporting of randomized clinical trials using a surgical intervention is in need of immediate improvement: a systematic review. Ann Surg 2006;244:677-83. 